\title{
Soil Mechanics Surface Sampler Experiment for Surveyor
}

\author{
RoNaLd F. ScOTT \\ Division of Engineering and Applied Science \\ California Institute of Technology, Pasadena
}

\begin{abstract}
The arrangement of an apparatus designed to measure the mechanical properties of the lunar surface from the Surveyor spacecraft is described. The experiments that may be made with this device are discussed and examples are given.
\end{abstract}

\section{INTRODUCTION}

One of the experimental devices that may be carried on the Surveyor spacecraft will measure the mechanical properties of the lunar surface in the vicinity of the spacecraft. The apparatus and the experiment to be performed are discussed, in addition to the interpretation of the results of the experiment. From the appearance and behavior of the lunar surface in the vicinity of Surveyor 1 [Jaffe et al., 1966], the experiment would be potentially fruitful in similar lunar areas.

\section{Apparatus}

The original 'surface sampler' was designed to obtain only samples of lunar surface material in the vicinity of the spacecraft. At a later stage in the development of Surveyor, the design of the surface sampler was revised to provide an instrument for measuring the mechanical properties of the lunar surface material.

The apparatus consists of an articulated mechanism (Figure i) that is capable of extending, contracting, swinging to the right or left in azimuth, and moving up or down in elevation. At the end of the mechanism is a scoop or bucket with a movable door. The door may be opened or closed, but it is not possible to select intermediate positions. The attitude of the apparatus is monitored by potentiometers attached to the shafts of the motors that control the motions of extension, azimuth, and elevation. Extension is powered by pretensioned springs mounted near the support of the sampler; they are arranged to drive the bucket outward. To draw it back, a retraction motor winds up a thin metal tape attached to the bucket.
In operation, the sampler arm may be raised and, by the actuation of a clutch, released to allow the bucket to fall on the lunar surface to break up any hard material or to reduce small rocks to more usable fragments. To increase the efficacy of the blow under lunar gravity, the downward motion is strengthened by means of a pretensioned torque spring attached to the elevation axis of the apparatus.

Two separate modes of command are provided, one to control with some precision the position of the surface sampler, and the other to permit relatively rapid movement of the sampler from one location to another. In the first mode, each motor is run for $0.1 \mathrm{sec}$; and, in the second mode, for 2 sec. The actual distance moved during each time interval depends on the motor employed and the load on it, but control is such that the bucket can be brought to within a few tenths of an inch of a selected position.

To make the apparatus capable of measuring the forces it applies to the lunar surface, three sensors were added: (1) a strain gage was bonded to one of the arms of the trellis to measure essentially the vertical component of force acting on the surface sampler; (2) another strain gage, attached near the bucket on a small cantilever arm to which the contraction tape is connected, measures the contraction force applied to the mechanism; and (3) an accelerometer was attached rigidly to the cutting edge of the bucket (Figure 2) to record the decelerations of the bucket on impact with the lunar surface.

The door of the bucket was also modified (Figure 2) to present, in the closed position at maximum extension, an area of about $13 \mathrm{~cm}^{2}$ $\left(2\right.$ in. $\left.{ }^{2}\right)$ to the lunar surface. In the open posi- 


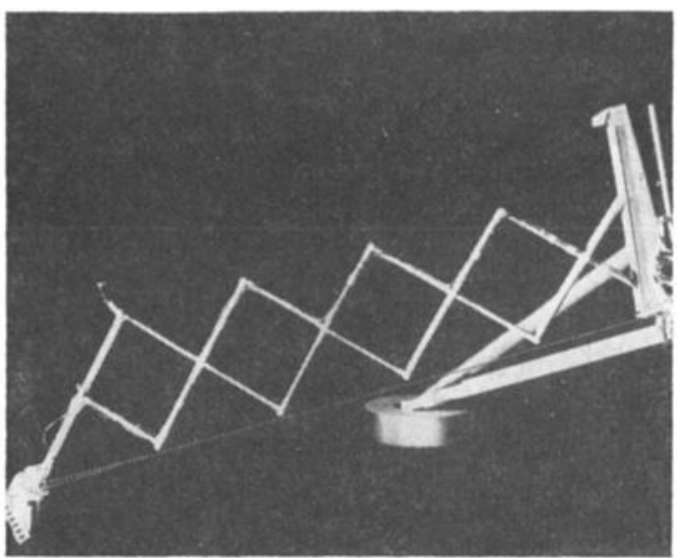

Fig. 1. The deployed surface sampler mounted on a mockup of the Surveyor spacecraft, one leg of which is seen in the background.

tion, the lower edge of the bucket, which may be placed in contact with the surface, has an area of about $0.5 \mathrm{~cm}^{2}\left(0.1 \mathrm{in}^{2}\right)$.

This instrumentation gives the surface sampler a very wide range of discrimination of the material properties of the lunar surface and permits quantitative determinations to better than 1 order of magnitude within a range of 6 orders of magnitude [Scott, 1965].

The instrumented surface sampler can perform the following surficial quantitative experiments:

1. Static vertical bearing or penetration test. The surface sampler is extended above the surface to a selected radius and the bucket is lowered by 0.1-sec increments until it makes contact with the lunar surface. On continuing the descent, the tip of the bucket is forced into the lunar soil. Throughout this sequence, the outputs of the vertical force strain gage and the elevation potentiometer are recorded. This information together with television pictures of the position of the surface sampler make it possible to obtain the force versus penetration record of the bucket as it is pushed into the surface. With a maximum downward force capability of about 1350 grams weight $(3 \mathrm{lb})$ at maximum extension, the maximum pressure that can be applied to the surface varies from a minimum of $100 \mathrm{~g} / \mathrm{cm}^{2}\left(1 \frac{1}{2} \mathrm{psi}\right)$, with the trap door closed, to about $2700 \mathrm{~g} / \mathrm{cm}^{2}$ (30 psi), with the door open.

2. Static drag or horizontal load test. After

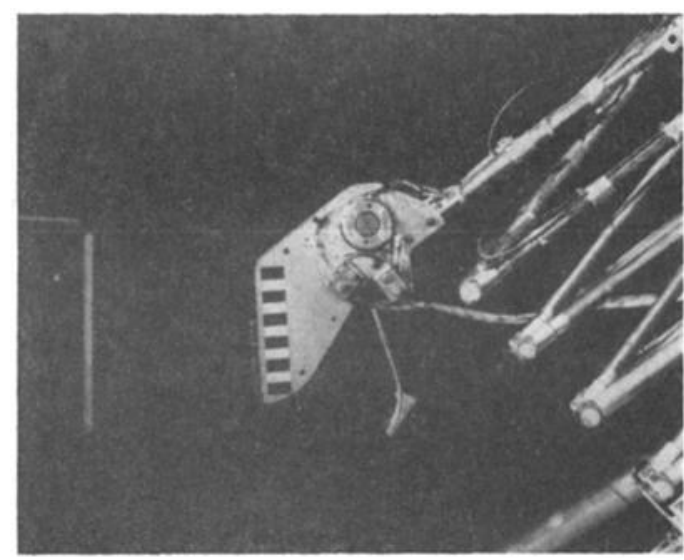

Fig. 2. The scoop or bucket and trap door. The accelerometer is mounted on the knife blade attached to the scoop.

a vertical loading test has been performed, the bucket may be pulled in toward the spacecraft on or through the surface material, and a measurement of the force exerted on the bucket can be made by means of the horizontal force strain gage output (Figure 3 ). This force includes both the force being applied to the lunar surface and the force required to contract the articulated mechanism itself. It will be necessary, therefore, to calibrate the contraction strain gage at the lunar surface by contracting the arm near the surface, but not in contact with it, while monitoring the force recorded by the strain gage. This will be done periodically during the Surveyor's life.

Successive drag tests at the same location can be employed to dig a trench. The surface sampler's geometrical arrangement and position on the spacecraft is such that theoretically

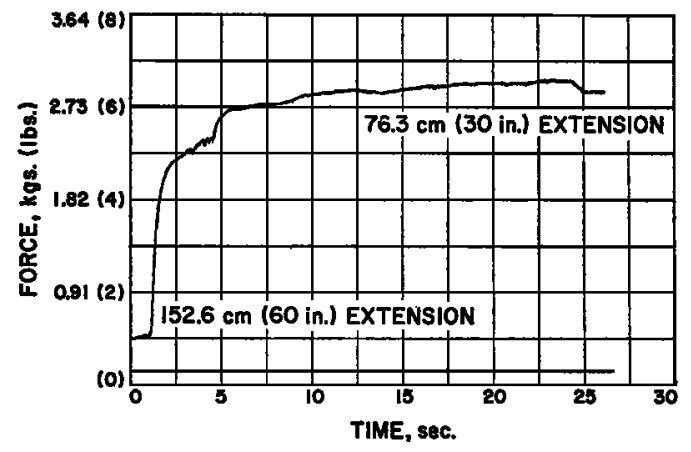

Fig. 3. The horizontal force measured during a surface sampler drag test in sand. 
a trench can be dug to a depth of about $45 \mathrm{~cm}$ (18 in.). Actually, the size of the trench will depend on the lunar surface properties and the power and time available for the trenching operation. At various stages in a trenching operation, vertical load penetration tests could be conducted to determine vertical inhomogeneity of the material.

Further information on the mechanical properties of the lunar surface will be obtained by photographic observation of the material's behavior during trenching and by study of the stability of the trench walls.

3. Impact deceleration test. When the surface sampler is dropped on the surface, the output of the accelerometer at the bucket end of the device can be obtained. This deceleration contains information on the lunar surface and on the vibration properties of the surface sampler arm itself. The interpretation of the record requires both studies of deceleration in various soils (Figures 4 and 5) and an examination of the vibration behavior of the surface sampler. Experiments [McCarty et al., 1964] have shown that the deceleration of an object hitting various materials has a characteristic form of deceleration as a function of time; this form is frequently called the deceleration 'signature.' The peak deceleration reached and the time for the deceleration to reach this peak are both dependent on the strength of the material impacted and the velocity of impact. In the case of the surface sampler, the velocity depends on the height from which it drops to the lunar surface. The high-frequency vibrations that are superimposed on the deceleration curve (Figure 4) are in themselves characteristic of the grain size or the vesicular structure of the material on which the impact occurs. Information on

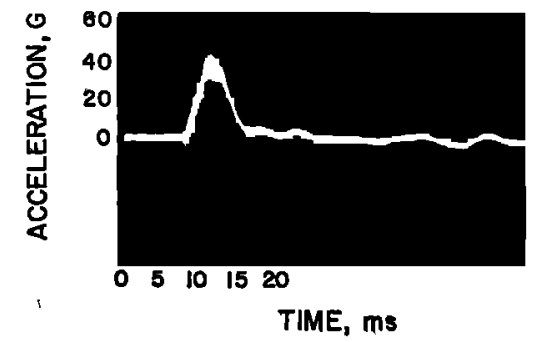

Fig. 4. The record of decleration obtained from an impact test $20.3-\mathrm{cm}$ ( 8 in.) drop in sand.

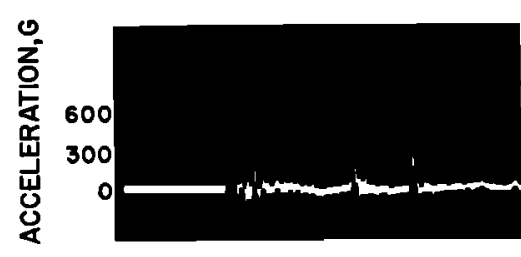

TIME, ms

Fig. 5. The record of deceleration obtained from an impact test $5.1-\mathrm{cm}$ ( 2 in.) drop on basalt.

these properties can be obtained from the acceleration record by passing the record through filters of different frequencies following the experiment.

In addition to the deceleration measurements, an impact experiment can give empirical information on the amount of force necessary to break small rocks or boulders on the surface, should any be accessible. The characteristics of the spring, surface sampler, and bucket are such that it is possible to break material of the hardness of a conventional building brick.

4. Weighing experiment. Since the vertical load can be monitored, it is possible to pick up and weigh a suitably sized pebble or rock fragment. If a television picture of the pebble is also possible, the dimensions of the pebble can be estimated by comparison with the scale painted on the side of the surface sampler (Figure 2); a value for the density of the pebble then can be computed to within 20 to $30 \%$ of its actual density. Although this is not sufficient to distinguish among many common rock materials on earth, it should be enough to determine whether the rock is solid or relatively porous. The distinction between the density of pumice and the density of an aggregate of grains would not, of course, be possible from this test.

5. Use in connection with $\alpha$-scattering experiment. The positions of the surface sampler and the deployed $\alpha$-scattering device [Turkevich et al., 1967] will be such that the surface sampler can pick up the $\alpha$-scattering device and place it in a new location on the lunar surface to provide more information on the atomic properties of the surface. The surface sampler also can clear off an area to a given depth to permit sensing of the subsurface material by the $\alpha$-scattering device. 


\section{Mode OF Operation}

The surface sampler should be capable of certain standard sequences of events in order to carry out the tests described above. For example, a normal sequence of events might consist of lowering the surface sampler bucket to the lunar surface at a preselected number of points and driving the bucket vertically downward through the surface to the limit of the apparatus's capability at each of these points. From the subsequent record, the 'mechanical surface' (the surface at which the sensitivity of the device first indicated a load) could be determined for comparison with the visual surface at the same point. In addition, a vertical load penetration test would have been carried out at each of these points. Following this, a drag test could be performed at selected cross sections, followed by a trenching operation to give information on the material below the surface.

Other standard sequences including, for example, drop tests, have been formulated and programmed. In practice, it may be desirable to select a program after television views of the actual surface in the vicinity of the spacecraft have been studied. After some preliminary tests, for example, an attempt to pick up, weigh, or break a rock may be of greater value than further standard tests.

Acknowledgments. The author is indebted to Mr. F. Roberson of the Jet Propulsion Laboratory of the California Institute of Technology for his assistance in formulating the experiments and carrying out the laboratory tests described in this paper.

The work described herein is performed under contract JPL-CIT 69811 with the Jet Propulsion Laboratory. The surface sampler was designed and is constructed by Hughes Aircraft Company, El Segundo, California.

\section{REFERENCES}

Jaffe, L. D., et al., Surveyor 1 : preliminary results, Science, 152, 1737-1750, June 24, 1966.

McCarty, J. L., A. G. Beswick, and G. W. Brooks, Application of penetrometers to the study of the physical properties of lunar and planetary surface, NASA Tech. Note D-2419, 1964.

Scott, R. F., Lunar problems in soil engineering, Proc. Am. Soc. Civil Engrs., J. Soil Mech. Found. Div., 91 , SM1, January 1965.

Turkevich, A. L., K. Knolle, E. Franzgrote, and J. H. Patterson, Chemical analysis experiment for the surveyor lunar mission, J. Geophys. Res., $72(2), 1967$.

(Received November 17, 1966.) 